\title{
FACTORED ARRANGEMENTS OF HYPERPLANES
}

\section{By Michel Jambu and Luis Paris}

Let $\mathbf{K}$ be a field and let $V$ be a vector space over $\mathbf{K}$. An arrangement of hyperplanes in $V$ is a finite family $\mathcal{A}$ of hyperplanes of $V$ through the origin. An arrangement $\mathcal{A}$ of hyperplanes is said to be real (resp. complex) if $\mathbf{K}=\mathbf{R}$ is the field of real numbers (resp. if $\mathbf{K}=\mathbf{C}$ is the field of complex numbers).

With an arrangement $\mathcal{A}$ of hyperplanes, one can associate a graded torsion-free $\mathbf{Z}$ algebra $A(\mathcal{A})$, called the Orlik-Solomon algebra of $\mathcal{A}$. If $\mathcal{A}$ is a complex arrangement, then $A(\mathcal{A})$ is isomorphic to the cohomology algebra of the complement

$$
M(\mathcal{A})=V-\left(\cup_{H \in \mathcal{A}} H\right)
$$

of $\mathcal{A}$ (see [OS1]). The Poincaré polynomıal $\operatorname{Poin}(\mathcal{A}, t)$ of $\mathcal{A}$ is the Poincaré polynomial of $A(\mathcal{A})$, namely,

$$
\operatorname{Poin}(\mathcal{A}, t)=\sum_{n=0}^{\infty} \operatorname{dim}\left(A^{n}(\mathcal{A})\right) t^{n}
$$

We refer to [Or] and [OT] for good expositions on the theory of arrangements of hyperplanes and, more precisely, on Orlik-Solomon algebras.

Let $\mathcal{A}$ be a real arrangement of hyperplanes. A chamber of $\mathcal{A}$ is a connected component of $V-\left(\cup_{H \in \mathcal{A}} H\right)$. We denote by $\mathcal{C}(\mathcal{A})$ the set of chambers of $\mathcal{A}$. For $C, D \in \mathcal{C}(\mathcal{A})$, we denote by $\mathcal{S}(C, D)$ the set of hyperplanes of $\mathcal{A}$ which separate $C$ and $D$. For a fixed chamber $C_{0} \in \mathcal{C}(\mathcal{A})$, we partially order $\mathcal{C}(\mathcal{A})$ by

$$
C \leq D \quad \text { if } \quad \mathcal{S}\left(C_{0}, C\right) \subseteq \mathcal{S}\left(C_{0}, D\right)
$$

$\mathcal{C}(\mathcal{A})$ provided with this order is denoted by $P\left(\mathcal{A}, C_{0}\right)$. It is a ranked bounded poset of finite rank, where $\operatorname{rank}(C)=\left|\mathcal{S}\left(C_{0}, C\right)\right|$ for $C \in \mathcal{C}(\mathcal{A})$. Its smallest element is $C_{0}$ and its greatest one is the chamber $-C_{0}$ opposite to $C_{0}$. The rank-generating function of $P\left(\mathcal{A}, C_{0}\right)$ is

$$
\zeta\left(P\left(\mathcal{A}, C_{0}\right), t\right)=\sum_{C \in \mathcal{C}(\mathcal{A})} t^{\operatorname{rank}(C)}
$$

The poset $P\left(\mathcal{A}, C_{0}\right)$ has been introduced and investigated by Björner, Edelman and Ziegler [Ed] [BEZ].

Let $\mathcal{A}$ be a real arrangement of hyperplanes. If $\mathcal{A}$ is either a supersolvable arrangement or a Coxeter arrangement, then there exist some integers $b_{1}, \ldots, b_{l}$ and a chamber $C_{0} \in \mathcal{C}(\mathcal{A})$ such that the Poincaré polynomial of $\mathcal{A}$ factors as

$$
\operatorname{Poin}(\mathcal{A}, t)=\prod_{i=1}^{l}\left(1+b_{i} t\right)
$$

Received June 19, 1993. 
(see [St] for supersolvable arrangements and [OS2] for Coxeter arrangements), the poset $P\left(\mathcal{A}, C_{0}\right)$ is a lattice (see [BEZ]), and the rank-generating function of $P\left(\mathcal{A}, C_{0}\right)$ factors as

$$
\zeta\left(P\left(\mathcal{A}, C_{0}\right), t\right)=\prod_{i=1}^{l}\left(1+t+\ldots+t^{b_{i}}\right)
$$

(see [BEZ] and [Pa] for supersolvable arrangements and [So] for Coxeter arrangements).

So, it is natural to ask whether there exists some relation between the Poincaré polynomial of a real arrangement $\mathcal{A}$ and the poset $P\left(\mathcal{A}, C_{0}\right)$ for some suitable chamber $C_{0} \in \mathcal{C}(\mathcal{A})$, and whether there exist other arrangements with such properties.

There is a class of arrangements of hyperplanes called free arrangements, introduced by Terao [Te1], and which contains supersolvable arrangements (see [JT]) and Coxeter arrangements (see [Te2]). If $\mathcal{A}$ is a free arrangement, then there exist some integers $b_{1}, \ldots, b_{l}$ such that the Poincaré polynomial factors as (1) (see [Te1]). However, if $\mathcal{A}$ is a free real arrangement of hyperplanes, then there does not necessarily exist a chamber $C_{0} \in \mathcal{C}(\mathcal{A})$ such that the rank-generating function of $P\left(\mathcal{A}, C_{0}\right)$ factors as (2); Terao [Te3] has found that the arrangement $A_{4}(17)$ from Grünbaum's list [Gr] is a counterexample.

Let $\mathcal{A}$ be an arrangement of hyperplanes. The intersection lattıce of $\mathcal{A}$ is the geometric lattice

$$
\mathcal{L}(\mathcal{A})=\left\{\bigcap_{H \in \mathcal{B}} H \mid \mathcal{B} \subseteq \mathcal{A}\right\},
$$

ordered by reverse inclusion. $V=\cap_{H \in \emptyset} H$ is the smallest element of $\mathcal{L}(\mathcal{A})$ and $\cap_{H \in \mathcal{A}} H$ is its greatest one. For $X \in \mathcal{L}(\mathcal{A})$, we set

$$
\begin{aligned}
& \mathcal{A}_{X}=\{H \in \mathcal{A} \mid H \supseteq X\}, \\
& \mathcal{A}^{X}=\left\{H \cap X \mid H \in \mathcal{A}-\mathcal{A}_{\boldsymbol{X}}\right\} .
\end{aligned}
$$

A partition $\Pi=\left(\Pi_{1}, \ldots, \Pi_{l}\right)$ of $\mathcal{A}$ into $l$ disjoint subsets is called independent if, for any choice of hyperplanes $H_{\imath} \in \Pi_{\imath}(i=1, \ldots, l)$, the rank of $H_{1} \cap \ldots \cap H_{l}$ is exactly $l$. If $X \in \mathcal{L}(\mathcal{A})$, then $\Pi$ induces a partition $\Pi_{X}$ of $\mathcal{A}_{X}$ which blocks are the nonempty subsets $\Pi_{\imath} \cap \mathcal{A}_{X}$. The partition $\Pi$ is a factorization (or a nıce partition) if

1) $\Pi$ is independent,

2) if $X \in \dot{\mathcal{L}}(\mathcal{A})-\{V\}$, then $\Pi_{X}$ has at least a block which is a singleton.

In particular, one of the blocks $\Pi_{i}$ is a singleton. We say that $\mathcal{A}$ is factored if $\mathcal{A}$ has a factorization.

Factored arrangements have been introduced and investigated by Jambu, Falk and Terao [JF] [Te4]. Supersolvable arrangements are factored (see [Ja]).

The homogeneous component $A^{1}(\mathcal{A})$ of degree 1 of the Orlik-Solomon algebra $A(\mathcal{A})$ of an arrangement $\mathcal{A}$ can be viewed as a free $\mathbf{Z}$-module spanned by the hyperplanes of $\mathcal{A}$. For $\mathcal{B} \subseteq \mathcal{A}$, we denote by $B(\mathcal{B})$ the submodule of $A^{1}(\mathcal{A})$ spanned by the hyperplanes included in $\mathcal{B}$. Following theorem is due to Terao [Te4].

ThEOREM 1 (Terao [Te4]). Let $\mathcal{A}$ be an arrangement of hyperplanes and let $\Pi=$ $\left(\Pi_{1}, \ldots, \Pi_{l}\right)$ be a partition of $\mathcal{A}$. The Orlik-Solomon algebra of $\mathcal{A}$, viewed as a graded Z-module, factors as

$$
A(\mathcal{A})=\left(\mathbf{Z} \oplus B\left(\Pi_{1}\right)\right) \otimes \ldots \otimes\left(\mathbf{Z} \oplus B\left(\Pi_{l}\right)\right)
$$


if and only if II is a factorization.

CoRollary 2 (Terao [Te4]). Let $\mathcal{A}$ be a factored arrangement of hyperplanes. Let $\Pi=\left(\Pi_{1}, \ldots, \Pi_{l}\right)$ be a factorization of $\mathcal{A}$.

1) The Poincaré polynomial of $\mathcal{A}$ factors as

$$
\operatorname{Poin}(\mathcal{A}, t)=\prod_{i=1}^{l}\left(1+\left|\Pi_{i}\right| t\right) .
$$

2) The multiset $\left\{\left|\Pi_{1}\right|, \ldots,\left|\Pi_{l}\right|\right\}$ depends only on $\mathcal{A}$.

3) For $X \in \mathcal{L}(\mathcal{A})$,

$$
\operatorname{rank}(X)=\left|\left\{i \mid \Pi_{i} \cap \mathcal{A}_{X} \neq \emptyset\right\}\right| .
$$

In partıcular, $l$ is the rank of $\mathcal{A}$.

Let $\mathcal{A}$ be a factored arrangement of hyperplanes and let $\Pi=\left(\Pi_{1}, \Pi_{2}, \ldots, \Pi_{l}\right)$ be a factorization of $\mathcal{A}$. We say that a hyperplane $H_{0} \in \mathcal{A}$ is distıngu a factorization $\Pi^{\prime}$ of $\mathcal{A}^{\prime}=\mathcal{A}-\left\{H_{0}\right\}$, namely, the nonempty subsets $\Pi_{\imath} \cap \mathcal{A}^{\prime}$ form a factorization of $\mathcal{A}^{\prime}$ (note that $\Pi_{2} \cap \mathcal{A}^{\prime}=\Pi_{\imath} \neq \emptyset$ if $H_{0} \notin \Pi_{i}$ ). Given a distinguished hyperplane $H_{0} \in \Pi_{1}$, we write $\Pi_{i}^{\prime \prime}=\left\{H \cap H_{0} \mid H \in \Pi_{i}\right\}$ for $i=2, \ldots, l$. We prove in [JP] the following result.

$$
\Pi^{\prime \prime}=\left(\Pi_{2}^{\prime \prime}, \ldots, \Pi_{l}^{\prime \prime}\right) \text { is a factorization of } \mathcal{A}^{\prime \prime}=\mathcal{A}^{H_{0}} \text {. }
$$

A factorization $\Pi=\left(\Pi_{1}, \ldots, \Pi_{l}\right)$ of an arrangement $\mathcal{A}$ of hyperplanes is said to be an inductıve factorization if there exists a distinguished hyperplane $H_{0} \in \mathcal{A}$ such that $\Pi^{\prime}$ is an inductive factorization of $\mathcal{A}^{\prime}=\mathcal{A}-\left\{H_{0}\right\}$ and $\Pi^{\prime \prime}$ is an inductive factorization of $\mathcal{A}^{\prime \prime}=\mathcal{A}^{H_{0}}$. We say that $\mathcal{A}$ is inductıvely factored if $\mathcal{A}$ has an inductive factorization.

A factored arrangement is not necessarily inductively factored, for example, the nine planes in $\mathbf{C}^{3}$ which satisfy the equation $\left(x^{3}-y^{3}\right)\left(x^{3}-z^{3}\right)\left(y^{3}-z^{3}\right)=0$ form a factored arrangement which is not inductively factored. Nevertheless, we do not know any arrangement $\mathcal{A}$ of hyperplanes in a real vector space which is factored and not inductively factored. Supersolvable arrangements are inductively factored.

In $[\mathrm{JP}]$ we prove the following results:

THEOREM 3. 1) Inductively factored arrangements are free.

2) Let $\mathcal{A}$ be an inductively factored arrangement of hyperplanes in a real vector space. Let $\Pi=\left(\Pi_{1}, \ldots, \Pi_{l}\right)$ be an inductive factorization of $\mathcal{A}$. There exists a chamber $C_{0} \in \mathcal{C}(\mathcal{A})$ such that $P\left(\mathcal{A}, C_{0}\right)$ is a lattice and its rank-generatıng function factors as

$$
\zeta\left(P\left(\mathcal{A}, C_{0}\right), t\right)=\prod_{i=1}^{l}\left(1+t+\ldots+t^{\left|\Pi_{i}\right|}\right)
$$

We do not know if 1) and 2) hold for any factored arrangement of hyperplanes.

An arrangement $\mathcal{A}$ of hyperplanes is essentıal if $\cap_{H \in \mathcal{A}} H=\{0\}$. Let $\mathcal{A}$ be an essential arrangement of hyperplanes in a $l$-dimensional real vector space $V=\mathbf{R}^{l}$. We provide $V$ with an arbitrary scalar product. Let $\mathbf{S}^{l-1}$ denote the unit sphere. The arrangement 
$\mathcal{A}$ determines a cellular decomposition (defined in Section 4) of $\mathbf{S}^{l-1}$ called the dual decomposition of $\mathbf{S}^{l-1}$ induced by $\mathcal{A}$.

THEOREM 4. If $\mathcal{A}$ has an inductive factorization $\Pi=\left(\Pi_{1}, \ldots, \Pi_{l}\right)$, then $\mathbf{S}^{l-1}$ can be viewed as the boundary $\partial \Omega$ of the l-cube

$$
\Omega=] 0,\left|\Pi_{1}\right|[\times \ldots \times] 0,\left|\Pi_{l}\right|[,
$$

and each cell of the dual decomposition of $\mathbf{S}^{l-1}=\partial \Omega$ has the form $I_{1} \times \ldots \times I_{l}$, where, for $i=1, \ldots, l$, either $I_{\imath}=\left\{a_{i}\right\}\left(a_{i} \in \mathbf{N}\right)$ or $\left.I_{i}=\right] a_{i}, b_{i}\left[\left(a_{i}, b_{i} \in \mathbf{N}\right)\right.$.

In [Sa] Salvetti associated with a real arrangement $\mathcal{A}$ of hyperplanes a cellular complex $\operatorname{Sal}(\mathcal{A})$ and proved that $\operatorname{Sal}(\mathcal{A})$ has the same homotopy type as the complement $M\left(\mathcal{A}_{\mathbf{C}}\right)$ of the complexification of $\mathcal{A}$. ¿From the dual decomposition of $\mathbf{S}^{l-1}$ induced by $\mathcal{A}$, we define a cellular decomposition of the (closed) unit disk $\mathbf{B}^{l}$ by attaching a $\mathbf{l}$-cell to $\mathbf{S}^{l-1}$. For every chamber $C \in \mathcal{C}(\mathcal{A})$, we fix a copy $\mathbf{B}(C)$ of $\mathbf{B}^{l}$ provided with this decomposition. The complex $\operatorname{Sal}(\mathcal{A})$ can be defined by

$$
\operatorname{Sal}(\mathcal{A})=\left\{\coprod_{C \in \mathcal{C}(\mathcal{A})} \mathbf{B}(C)\right\} / \sim
$$

where $\sim$ is an equivalence relation which, for each pair $(C, D)$ of chambers, identifies some cells of $\mathbf{B}(C)$ with their corresponding cells of $\mathbf{B}(D)$. So, Theorem 4 may be certainly used to investigate the homotopy of the complement $M\left(\mathcal{A}_{\mathbf{C}}\right)$ of the complexification of an inductively factored arrangement $\mathcal{A}$.

Let $\mathcal{A}$ be a real arrangement of hyperplanes. Assume that $\mathcal{A}$ has a factorization $\Pi=\left(\Pi_{1}, \ldots, \Pi_{l}\right)$. For $C, D \in \mathcal{C}(\mathcal{A})$ and $i \in\{1, \ldots, l\}$, we set $\mathcal{S}_{i}(C, D)=\mathcal{S}(C, D) \cap \Pi_{\imath}$. For $b \in \mathbf{N}$, we write $[b]=\{0,1, \ldots, b\}$. The counting map of $\mathcal{A}$ with respect to a chamber $C_{0} \in \mathcal{C}(\mathcal{A})$ is the morphism $\phi\left(\Pi, C_{0}\right)$ of ranked posets defined by

$$
\begin{array}{ccc}
\phi\left(\Pi, C_{0}\right): P\left(\mathcal{A}, C_{0}\right) & \longrightarrow c & {\left[\left|\Pi_{1}\right|\right] \times \ldots \times\left[\left|\Pi_{l}\right|\right]} \\
C & \longmapsto\left(\left|\mathcal{S}_{1}\left(C_{0}, C\right)\right|, \ldots,\left|\mathcal{S}_{l}\left(C_{0}, C\right)\right|\right)
\end{array}
$$

where $\left[\left|\Pi_{1}\right|\right] \times \ldots \times\left[\left|\Pi_{l}\right|\right]$ is partially ordered by

$$
\left(a_{1}, \ldots, a_{l}\right) \leq\left(b_{1}, \ldots, b_{l}\right) \text { if } a_{1} \leq b_{1}, \ldots, a_{l} \leq b_{l}
$$

and, for $\left(a_{1}, \ldots, a_{l}\right) \in\left[\left|\Pi_{1}\right|\right] \times \ldots \times\left[\left|\Pi_{l}\right|\right]$,

$$
\operatorname{rank}\left(a_{1}, \ldots, a_{l}\right)=a_{1}+\ldots+a_{l} .
$$

Note that, in general, the counting map $\phi\left(\Pi, C_{0}\right)$ is not an isomorphism of ranked posets, even if $\phi\left(\Pi, C_{0}\right)$ is a bijection; two chambers $C$ and $D$ may be not comparable meanwhile their images are comparable.

Let $\Pi=\left(\Pi_{1}, \ldots, \Pi_{l}\right)$ be a factorization of $\mathcal{A}$. We say that $\Pi$ is a hyperfactorization if there exists a chamber $C_{0} \in \mathcal{C}(\mathcal{A})$ such that $\phi\left(\Pi, C_{0}\right)$ is a bijection. We say that $\mathcal{A}$ is hyperfactored if $\mathcal{A}$ has a hyperfactorization.

\section{THEOREM 5. Inductively factored real arrangements are hyperfactored.}

Most of the results on inductively factored arrangements mentioned above actually are proved for hyperfactored arrangements. We do not know any real arrangement $\mathcal{A}$ 
which is hyperfactored and not inductively factored. In fact, we introduce this notion of "hyperfactorization" for technical reasons, indeed, in order to prove that inductively factored arrangements are hyperfactored, we need several preliminary results on hyperfactored arrangements. So, all results stated in this paper for hyperfactored arrangements hold for inductively factored arrangements.

Example. Let $\mathcal{A}$ be the arrangement in $\mathbf{R}^{3}$ projectively pictured in Figure $1(\mathcal{A}$ contains the "line" at infinity). Set $\Pi_{1}=\left\{H_{1}, H_{3}, H_{4}\right\}, \Pi_{2}=\left\{H_{2}, H_{5}, H_{6}\right\}$, and $\Pi_{3}=$ $\left\{H_{7}\right\}$. Then $\Pi=\left(\Pi_{1}, \Pi_{2}, \Pi_{3}\right)$ is a factorization of $\mathcal{A}$. One can easily verify that $H_{1}$ is distinguished in $\mathcal{A}, H_{2}$ is distinguished in $\mathcal{A}-\left\{H_{1}\right\}, H_{3}$ is distinguished in $\mathcal{A}-\left\{H_{1}, H_{2}\right\}$, and so on. Hence, $\Pi$ is an inductive factorization.

The adjacency graph of $\mathcal{A}$ is defined to be the graph which vertices are the chambers of $\mathcal{A}$, and where two chambers are joined by an edge if they are adjacent. It is shown in Figure 2. From this graph, one can verify that $\phi\left(\Pi, C_{0}\right)$ is a bijection from $\mathcal{C}(\mathcal{A})$ to $[3] \times[3] \times[1]$, thus $\Pi$ is a hyperfactorization. Moreover, $P\left(\mathcal{A}, C_{0}\right)$ is a lattice and

$$
\zeta\left(P\left(\mathcal{A}, C_{0}\right), t\right)=\left(1+t+t^{2}+t^{3}\right)^{2}(1+t) .
$$

We set $\Omega=] 0,3[\times] 0,3[\times] 0,1\left[\right.$. The dual decomposition of $\mathbf{S}^{2}=\partial \Omega$ induced by $\mathcal{A}$ is shown in Figure 3.

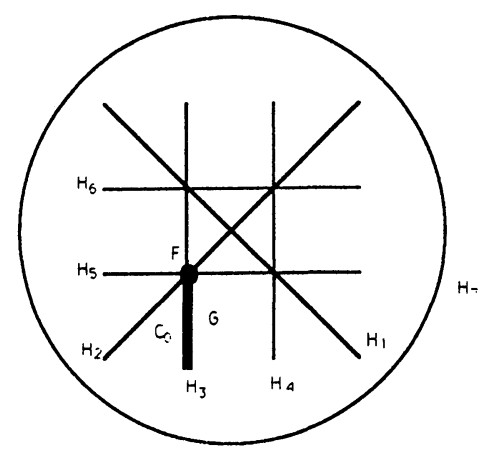

Figure 1

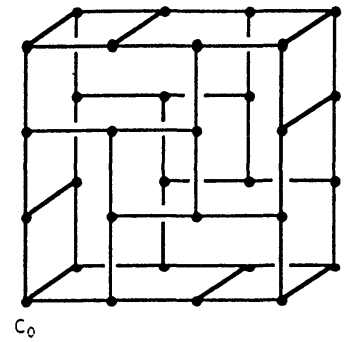

Figure 2

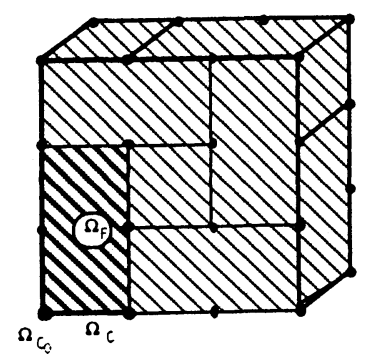

Figure 3

Our paper [JP] is organized as follows.

In Section 2 we mainly prove that, if $\mathcal{A}$ is a factored arrangement of hyperplanes and $H_{0} \in \mathcal{A}$ is distinguished, then $\mathcal{A}^{\prime \prime}=\mathcal{A}^{H_{0}}$ is factored. We also prove that inductively factored arrangements are free. 
In Section 3 we prove several results on hyperfactored arrangements. In particular, we characterize the chambers $C_{0} \in \mathcal{C}(\mathcal{A})$ such that $\phi\left(\Pi, C_{0}\right)$ is a bijection, we prove there are $2^{\operatorname{rank}(\mathcal{A})}$ of them, we prove that, for such a chamber $C_{0}$, the rank-generating function of $P\left(\mathcal{A}, C_{0}\right)$ factors as

$$
\zeta\left(P\left(\mathcal{A}, C_{0}\right), t\right)=\prod_{i=1}^{l}\left(1+t+\ldots+t^{\left|\Pi_{i}\right|}\right),
$$

and, if $C$ is a chamber of a hyperfactored arrangement $\mathcal{A}$, then $C$ has at most $2 \cdot \operatorname{rank}(\mathcal{A})-$ 1 walls.

In Section 4 we prove that the dual decomposition of the sphere $\mathbf{S}^{l-1}$ induced by a hyperfactored arrangement can be viewed as the decomposition of the boundary $\partial \Omega$ of the $l$-cube $\Omega$ described above.

In Section 5 we prove that, if $\mathcal{A}$ is a hyperfactored arrangement and $C_{0} \in \mathcal{C}(\mathcal{A})$ is a suitable chamber, then the poset $P\left(\mathcal{A}, C_{0}\right)$ is a lattice.

In Section 6 we prove that inductively factored real arrangements are hyperfactored.

ACKnowledgement: The first author is grateful to all the organizers of this Workshop for their hospitality and he would like to thank more particularly Professor Huynh Mui, Professor Yukio Matsumoto and Professor Mutsuo Oka.

\section{REFERENCES}

[BEZ] A. BJöRNER, P.H. EDELMAN and G.M. ZIEGLER, Hyperplane arrangements with a lattice of regıons, Discrete Comput. Geom. 5 (1990), 263-288.

[Ed] P.H. Edelman, A partial order on the regıons of $\mathbf{R}^{n}$ dissected by hyperplanes, Trans. Amer. Math. Soc. 283 (1984), 617-631.

[FJ] M. FALK and M. JAMBU, Factorizations and colorings of combınatorial geometries, preprint.

[Gr] B. Grünbaum, Arrangements and spreads, AMS Regional Conference Series in Mathematics, Vol. 10, Amer. Math. Soc., Providence, 1972.

[Ja] M. JAMBU, Fiber-type arrangements and factorızation properties, Advances in Math. 80 (1990), $1-21$.

[JP] M. JAMBU and L. PARIS, Combınatorıcs of ınductively factored arrangements, to appear in European J. of Combinatorics.

[JT] M. JAMBU and H. TERAO, Free arrangements of hyperplanes and supersolvable lattices, Advances in Math. 52 (1984), 248-258.

[LW] A.T. LUNDell and S. Weingram, "The topology of CW complexes," Van Nostrand Reinhold Company, New York, 1969.

[Or] P. ORLIK, "Introduction to arrangements," CBMS Lecture Notes 72, Amer. Math. Soc., 1989.

[OS1] P. ORLIK and L. SOLOMON, Combinatorics and topology of complements of hyperplanes, Invent. Math. 56 (1980), 167-189.

[OS2] P. ORLIK and L. SOLOMON, Unitary reflection groups and cohomology, Invent. Math. 59 (1980), 77-94.

[OT] P. ORLIK and H. TeraO, "Arrangements of hyperplanes," Springer-Verlag, New York, 1992.

[Pa] L. PARIS, The counting polynomial of a supersolvable arrangement, Advances in Math., to appear.

[Sa] M. SALVETTI, Topology of the complement of real hyperplanes in $\mathbf{C}^{N}$, Invent. Math. 88 (1987), 603-618.

[So] L. Solomon, The orders of the finte Chevalley groups, J. Algebra 3 (1966), 376-393. 
[Sp] E.H. Spanier, "Algebraic topology," Mc Graw Hill Book Company, New York, 1966.

[St] R. StANLEY, Supersolvable lattices, Algebra Universalis 2 (1972), 197-217.

[Te1] H. TERAO, Arrangements of hyperplanes and theır freeness I, II, J. Fac. Sci. Univ. Tokyo 27 (1980), 293-320.

[Te2] H. TERAO, Free arrangements of hyperplanes and unitary reflection groups, Proc. Japan Acad. Ser. A 56 (1980), 389-392.

[Te3] H. TERAO, letter to A. Björner, August 21th, 1986.

[Te4] H. TERAO, Factorizations of Orlik-Solomon algebras, Advances in Math 91 (1992), 45-53.

[Za] T. ZASLAVSKY, "Facing up to arrangements: Face-count formulas for partitions of space by hyperplanes," Memoirs Amer. Math. Soc. No. 154, 1975.

\author{
MiChEL JAMBU \\ DÉPARTEMENT DE MATHÉMATIQUES \\ UNIVERSITÉ DE NANTES \\ 2 RUE DE LA HOUSSINIÈRE \\ 44072 Nantes Cedex 03 \\ FRANCE \\ e-mail: jambu@namath.dnet.circe.fr \\ LUIS PARIS \\ DÉPARTEMENT DE MATHÉMATIQUES \\ UNIVERSITÉ DE BOURGOGNE \\ B.P. 138 \\ 21004 Dijon Cedex \\ FRANCE \\ e-mail: lparis@satie.u-bourgogne.fr
}

